\title{
A Model of Mesons based on $\chi S B$ in the Light-Front Frame
}

\author{
L. Susskind \\ Physics Department, Stanford University \\ Stanford, CA 94309 \\ M. Burkardt \\ Institute for Nuclear Theory \\ University of Washington \\ Seattle, WA 98195
}

\section{PREPARED FOR THE U.S. DEPARTMENT OF ENERGY UNDER GRANT DE-FG06-90ER40561}

This report was prepared as an account of work sponsored by the United States Government. Neither the United States nor any agency thereof, nor any of their employees, makes any warranty, express or implied, or assumes any legal liability or responsibility for the accuracy, completeness, or usefulness of any information, apparatus, product, or process disclosed, or represents that its use would not infringe privately owned rights. Reference herein to any specific commercial product, process, or service by trade name, mark, manufacturer, or otherwise, does not necessarily constitute or imply its endorsement, recommendation, or favoring by the United States Government or any agency thereof. The views and opinions of authors expressed herein do not necessarily state or reflect those of the United States Government or any agency thereof. 


\section{DISCLAIMER}

Portions of this document may be illegible in electronic image products. Images are produced from the best available original document. 


\title{
A Model of Mesons based on $\chi \mathrm{SB}$ in the Light-Front Frame ${ }^{1}$
}

\author{
L. Susskind \\ Physics Department, Stanford University \\ Stanford, CA 94309 \\ M. Burkardt \\ Institute for Nuclear Theory, University of Washington \\ Seattle, WA 98195
}

\begin{abstract}
Spontaneous breaking of chiral symmetry is discussed in the light-cone framework. The essential ingredient is an infinite number of constituents near zero light-cone momentum. These high (light-cone) energy degrees of freedom freeze out and leave behind some explicit symmetry breaking in the low (light-cone) energy effective Hamiltonian. Connections with Regge theory and soft pion theorems are discussed. Taking the order parameter to be the 4-th component of a chiral 4 -vector, the effect of the spontaneous symmetry breaking on meson masses and decay width is calculated and compared with experimental data.
\end{abstract}

\section{Introduction}

Long before QCD, when I started thinking about field theory in the light-cone (LC) frame [1], I was attracted by the fact that in the LC frame the vacuum is just empty space and there is no confusion between vacuum and particles. Since I was trying to understand hadrons, it seemed unnecessary to spend time understanding the vacuum - and getting out "absolutely nothing". Of course, I was wrong and the vacuum is very interesting, but it still sounds like a nice idea to calculate hadrons in the infinite momentum frame [2], where everything moves so fast relative to the vacuum that it would decouple from it in some sense.

Around 1972, i.e. when QCD was considered as a candidate for the strong interactions but before QCD became the standard understanding of what the strong interaction was, it became fairly clear that the basic problems of strong interaction was twofold: the confinement problem and the chiral symmetry breaking problem. Since I was more interested in confinement, and since the LC frame does not seem to be an especially helpful way to

\footnotetext{
${ }^{1}$ Based on a lecture given by L. Susskind at the workshop on 'Theory of Hadrons and Light-Front QCD' at Polona Zgorzelisko, Poland, August 15-25,1994.
} 
think about confinement, I abandoned the LC and started thinking about lattice gauge theory. But I also felt that chiral symmetry breaking $(\chi \mathrm{SB})$ is a problem that could very well be understood in the LC frame. First it seems a bit of a puzzle how a symmetry breaking can occur in the LC frame because, after all, in the LC frame the vacuum is just the structureless Fock space vacuum. In this lecture, I will provide a picture of how $\chi \mathrm{SB}$ can occur in the LC framework and I will work out some of the consequences.

The parton model is an interesting way to think about hadrons. In the naive parton model, one simply pictures a fast moving particle as being some collection of constituents with relatively large momentum, such that when one boosts the system, doubles its momentum, all these partons double their momenta and so forth. Upon boosting the system to infinite momentum the partons would all become very far from $\eta=0$, where $\eta$ is the fraction of the particle's longitudinal momentum carried by the parton. Since all the vacuum activity takes place at $\eta=0$, it seems very curious how these partons (at finite $\eta$ ) could "feel" what is going on at $\eta=0$.

Chiral symmetry is the symmetry generated by $Q_{5}^{\alpha}$, where $\alpha$ is some isospin index. In the LC frame, this is just the sum over all partons of the parton's helicity times its isospin times a plus or a minus - depending on whether it is a quark or an antiquark [5]. Therefore the conservation of chiral symmetry in the LC frame is a very simple thing: it just says that some kind of "generalized helicity" is conserved and it does not appear to have to do with quark masses. Since quark masses can be introduced into the LC frame without causing problems about helicity conservation, one encounters the next puzzle: how come that if one gives a mass to a quark, chiral symmetry is broken in an ordinary frame of reference but in the LC frame it is not? Besides being confusing this is also a nuisance because obviously one cannot break chiral symmetry by simply adding an "induced mass term" to the LC Hamiltonian.

The right way to think about spontaneous breaking of chiral symmetry on the LC is that it somehow manifests itself through interactions between partons at finite $\eta$ and $\eta=0$ (the vacuum). The problem or puzzle with this is that matrixelements connecting states which are separated by a large distance in rapidity ${ }^{2}$ are suppressed. So ho how could the valence quarks possibly feel what is going on at $\eta=0$ ? After QCD was invented, there seemed to be a mechanism by which things at finite $\eta$ can connect to $\eta=0$, namely by emitting a soft gluon. Since the spin of gluons is larger than that of quarks they can more easily connect regions that are widely separated in rapidity. However, the emission of a soft gluon does not flip the helicity of the quark and therefore it does not seem that gluons being emitted into the $\eta=0$ region has much to do with the $\chi \mathrm{SB}$.

Before we embark on constructing a model for spontaneous symmetry breaking, I should emphasize some very fundamental property of LC Hamiltonians: under a rescaling of the LC momentum, $\eta \rightarrow \lambda \eta$, the LC-Hamiltonian scales like $H \rightarrow H / \lambda$. This looks like a dilatation symmetry if we think of the $\eta$-axis as a spatial axis. Of course, it is

\footnotetext{
${ }^{2}$ Rapidity is the logarithm of the LC-momentum $\eta$.
} 
not a spatial axis, but if we assume that things are short range coupled on the $\eta$-axis we can consider it as if it were a spatial axis and formulate a field theory on this axis. The dilatation symmetry then reflects some underlying scale invariance of this field theory and the right tool for studying such a system is the renormalization group.

\section{The Long Arm of the Vacuum ${ }^{3}$}

A particle or hadron is not just a collection of partons at finite $\eta$. In fact, partons fill the $\eta$-axis in a way which gets denser and denser as one goes to smaller $\eta$ : according to Feynman and Bjorken, the number of constituents per unit $\eta$ is given by $d \eta / \eta$. The resulting accumulation of partons at low $\eta$ is usually called the wee partons. The question here is: can one imagine a process that would allow to transmit the information about the $\chi \mathrm{SB}$ from $\eta=0$ through the chain of wee partons to finite $\eta$; one at a time, so that at no place large momentum is transferred.

At first this seems impossible: if one thinks of the $\eta$-axis as being more or less short range coupled (short "range" in $\eta$ space) then one can regard the wee partons as some kind of $1+1$ dimensional system. Since one cannot have spontaneous symmetry breaking of a continuous symmetry in a $1+1$ dimensional system, this seems forbidden right away. However, as the following model demonstrates, there is in fact no problem about the $1+1$ dimensionality. Let us consider a collection of constituents which are distributed along the $\eta$-axis according to $d \eta / \eta$. Each constituent is assumed to have a LC momentum $\eta_{i}$ and some internal degree of freedom. For simplicity, we will consider a $U(1)$ symmetry here, which will not be specified any further. The $U(1)$ phase of each parton will be denoted by $\phi_{i}$ with conjugate momentum $\Pi_{\phi_{i}}$. The Hamiltonian is assumed to consist of a kinetic term, $\Pi_{\phi_{i}}^{2} / \eta_{i}$, for each constituent plus a nearest neighbor (in $\eta$-space) coupling of the form $\left(\phi_{i}-\phi_{i-1}\right)^{2} / \bar{\eta}$, where $\bar{\eta}$ can be chosen to be the average $\eta$ of the two constituents. The $\eta$-dependence of these terms has been chosen such that the LC-Hamiltonian scales like LC-momentum ${ }^{-1}$. One obtains

$$
H=\sum_{i}\left(\frac{\Pi_{\phi_{i}}^{2}}{\eta_{i}}+g \frac{\left(\phi_{i}-\phi_{i-1}\right)^{2}}{\eta_{i}+\eta_{i+1}}\right) .
$$

The $d \eta / \eta$ distribution of partons can be built into this model by furthermore assuming that $\Delta \eta$, the distance between neighboring partons, goes like $\varepsilon \eta$, where $\varepsilon$ is some small parameter, characterizing the density of partons on the rapidity axis. This Hamiltonian can be solved by a simple mathematical trick. For small $\varepsilon$, one can make a continuum approximation, and the Hamiltonian becomes

$$
H=\int_{0}^{1} d \eta\left(\Pi_{\phi_{\eta}}^{2}+g\left(\nabla_{\eta} \phi_{\eta}\right)^{2}\right),
$$

\footnotetext{
${ }^{3}$ There is an expression in English the long arm of the law which means you cannot get away from the police, no matter how hard you try.
} 
where we have arbitrarily picked $\eta=1$ as the endpoint of the axis. A nice order parameter for the $U(1)$ symmetry of this system is $\cos \phi$. Since Eq.(2) is just the Hamiltonian for a free massive field, one finds that $\langle 0|\cos \phi| 0\rangle$ is essentially given by $\exp \left(-\left\langle 0\left|\phi^{2}\right| 0\right\rangle\right)$ (this result is obtained by expanding $\cos \phi$ and calculating all the contractions). Usually in quantum field theory, the expectation value of the square of a field is infinite (in this case it would be logarithmically infinite). Therefore this kind of matrix element usually vanishes. Of course this is an UV-divergence and the theory should be cut off. A natural cutoff is provided by $\Delta \eta=\varepsilon \eta$, the spacing between neighboring constituents before we made a continuum approximation of the system and hence $\varepsilon$ plays the role of some kind of UV-regulator here. With such a cutoff in place one obtains

$$
\left\langle 0\left|\phi_{\eta}^{2}\right| 0\right\rangle \sim \log \frac{\eta}{\Delta \eta}=\log \frac{1}{\varepsilon},
$$

and as long as $\varepsilon$ is finite, i.e. as long as the density of partons on the rapidity axis is not infinite, one gets a finite nonvanishing matrix element for the order parameter $\langle 0|\cos \phi| 0\rangle$. Why does this violate the usual rules about $1+1$ dimensions? Usually, what happens is $1+1$ dimensional systems fluctuate too strongly such that there is no order left. Here the coupling, i.e. the spring constant between neighboring partons, gets stronger and stronger as one approaches $\eta=0$ so rapidly that the system is able to hold itself together - despite the fact that there is an infinite number of steps between $\eta=0$ and finite $\eta$. It thus becomes possible to have spontaneous symmetry breaking.

\section{Regge Theory}

Let us consider now the spatial distribution of the partons in the transverse directions. In principle, there is some wavefunction from which one can calculate this distribution. However, many qualitative features can be understood on the basis of very simple arguments. First let us order the partons in sequence of rapidity. We will again assume that things are short range coupled in rapidity. Therefore, if we go down the chain in $\eta$, the transverse position behaves on average like a random walk, i.e. its square grows like the number of partons down the chain

$$
r_{\perp}^{2} \propto-\log \eta
$$

Assuming that the transverse spatial distribution at position $\eta$ is Gaussian, $\rho\left(\vec{r}_{\perp}^{2}, \eta\right) \sim$ $\exp \left(-\vec{r}_{\perp}^{2} / \log \eta\right)$, one can compute the Fourier transform

$$
F\left(\vec{q}_{\perp}\right) \sim \exp \left(-\vec{q}_{\perp}^{2} \log \eta\right)=\eta^{-\vec{q}_{\perp}^{2}} .
$$

Of course, there are all kinds of dimensionful constants which have been left out in these equations. 
This formula (5) is a special case of a very general feature, namely that many quantities have power law dependence on $\eta$. The reason power laws keep reoccuring is because of the abovementioned scale invariance. The formal machinery which is usually being used to describe this behavior is Regge pole theory.

As I have indicated already, other quantities are also likely to be power law distributed. Let us consider the average charge per parton. For small $\eta$ it has to vanish - otherwise the total charge carried by the partons is infinite. Experimentally, one obtains for the average charge per parton at position $\eta$ approximately

$$
e(\eta) \approx \hat{e} \eta^{0.5} \text {. }
$$

$\hat{e}$ is called the residue. Now let us combine this result with the transverse distribution of the partons to calculate the electromagnetic form factor of the hadron. One obtains

$$
F^{E M}\left(\vec{q}_{\perp}\right)=\int_{0}^{1} \frac{d \eta}{\eta} e(\eta) \eta^{-\vec{q}_{\perp}^{2}}=\frac{\hat{e}}{\vec{q}_{\perp}^{2}+1 / 2},
$$

which represents a particle pole at $m^{2}=1 / 2$ and coupling $\hat{e}$. Very generally, there is a connection between these power laws and the spectrum of particles coupling to the hadron. So the two elements of the theory are

- that one can think of the $\eta$-axis as an axis on which one can do field theory and talk about distributions

- that the spatial distribution of partons satisfy certain assumptions, which allow one to compute particle masses and couplings in terms of these distributions.

It should be emphasized that the exponents usually do in general not depend on the hadron under consideration (the residues do very well). Furthermore, the above discussion can be repeated for off-diagonal matrix elements (i.e. transition amplitudes) in which case the residues assume some kind of matrix structure, i.e. they can be considered as operators acting between hadronic states.

\section{4. $\chi \mathrm{SB}$ and the Pion}

As long as the rapidity axis is sufficiently short range coupled, one can, in addition to densities on the $\eta$-axis (above we have already discussed the charge density), also introduce currents and, at least in the case of conserved charges, there should also be continuity equations, $\dot{\rho}+d j / d \eta=0$. In the following we will apply these results to the axial current, which can flow up and down the $\eta$-axis, but which is more or less locally conserved. For this purpose, let me introduce a chiral 4 -vector $\phi_{\alpha}$ on the $\eta$-axis. Note that $\alpha$ is NOT a space time index but is related to $(\vec{\pi}, \sigma)$. The 4 -th component of $\phi_{\alpha}$, 
basically $\sigma$, represents an order parameter for $\chi \mathrm{SB}$. These chiral 4 -vectors tend to line up exactly as if they were a ferromagnet. Therefore they will be called chiral magnets in the following. The point is that the couplings between all these chiral magnets goes like $1 / \eta$, i.e. the low $\eta$ degrees of freedom are extremely frozen. That is characteristic of the LC-frame. Therefore, if we bring in one "external" magnet, it will not be able to upset this order. It will precess, like a spin in an external magnetic field, and chiral charge will flow in and out from the region near the external probe.

So let us assume now that there is a nonvanishing axial charge density $j_{5}$ at small $\eta$ and demonstrate that this implies a massless pion. Furthermore, we will see that this massless pion couples to the axial current in a particular way which allow one to make models in which one can calculate properties of the pion and of the hadron spectrum. First let us take the chirally symmetric Hamiltonian and add some small term which explicitly breaks the chiral symmetry

$$
H=H_{s y m m}+c \int_{0}^{1} \frac{d \eta}{\eta} \phi_{4}(\eta)
$$

where $c$ is some small constant. A straightforward calculation yields

$$
-i \dot{Q}_{\alpha 5}=\left[H, Q_{\alpha 5}\right]=c \int_{0}^{1} \frac{d \eta}{\eta^{2}} \phi_{\alpha}(\eta)
$$

where $\alpha$ runs from 1 to 3 .

In order to derive some observable consequences of the above picture, let us assume that the matrix elements of $\phi_{\alpha}$ are also power law behaved, i.e.

$$
\phi_{\alpha}(\eta) \sim \hat{\phi}_{\alpha} \eta^{1+\mu}
$$

where $\mu$ is some number. This yields

$$
-i \dot{Q}_{\alpha 5}=c \hat{\phi} \int_{0}^{1} \frac{d \eta}{\eta^{2}} \eta^{1+\mu}=\frac{c \hat{\phi}}{\mu} .
$$

The only way to keep $\dot{Q}_{\alpha 5}$ from vanishing when $c \rightarrow 0$, is to let $\mu \rightarrow 0$ linearly at the same time. In order to avoid having too many constants in this discussion, let me just assume $\mu=c$. The next step is to use the knowledge of how $\phi$ has to behave to compute a formfactor for something which couples to $\phi_{\alpha}$. The calculation more or less parallels the calculation of the electromagnetic case above except that the $1 / 2$ gets replaced by $c$ and one obtains a formfactor which goes like $\hat{\phi} /\left(\vec{q}_{\perp}^{2}+c\right)$, indicating a particle pole at $c$. Due to the (small) explicit symmetry breaking, the pion has a mass $c$ and it becomes massless in the "chiral" limit $(c \rightarrow 0)$. Furthermore we found that the matrix element of $\dot{Q}_{\alpha 5}$ is, up to some numerical constants, equal to the emission amplitude of a pion. 
So far we have established that within a parton model with short range couplings on the rapidity axis one can define, chiral charges and order parameter. We have furthermore obtained a basic equation for the on shell pion emission amplitude ${ }^{4}$

$$
T_{A B}^{\pi}=\frac{2}{i f_{\pi}}\left\langle A\left|\dot{Q}_{\alpha 5}\right| B\right\rangle=-\frac{2}{f_{\pi}}\left(M_{A}^{2}-M_{B}^{2}\right)\left\langle A\left|Q_{\alpha 5}\right| B\right\rangle,
$$

which is a generalization of the Goldberger-Treiman relation.

\section{Effective Hamiltonians}

On the $\eta$-axis we have degrees of freedom, which get more and more strongly coupled as go down towards $\eta=0$. Usually in physics when we encounter problems with higher and higher energy scales, we cut off the theory and make an effective theory. Instead of studying the whole chain, we will try cut off the highest frequency parts, by introducing a cutoff $\varepsilon$ on $\eta$. In the presence of the cutoff, the scale invariance mentioned in section 2 is broken. However, we still want the physics to be independent of the artificial parameter $\varepsilon$. Therefore, when we construct the effective Hamiltonian, we have to look for a consistency of descriptions as we move the cutoff further and further away, while keeping the physics fixed. In other words, what we have to look for is an UV fixed point for the renormalization group transformations of this system.

The Hamiltonian evidently consists of three pieces in general. One of them, $H_{>\varepsilon}$, will have to do with the degrees of freedom above the cutoff. The of course there will be a part $H_{<\varepsilon}$. Furthermore, there will be a term $H_{\varepsilon}$ which couples the degrees of freedom in these two regions. First of all, the degrees of freedom governed by $H_{<\varepsilon}$ are high energy degrees of freedom and it costs a large amount of energy to disrupt them. ${ }^{5}$ Therefore $H_{<\varepsilon}$ is effectively just a number. Similarly, $H_{\varepsilon}$, to the extend that it depends on degrees of freedom in the frozen region, one can also use vacuum expectation values (VEVs). Of course for modes with $\eta>\varepsilon$ one must keep the full operator structure in $H_{\varepsilon}$. Now since this system is frozen into a chirally asymmetric configuration, it is very natural to assume that in replacing the $\eta<\varepsilon$ modes by their VEV in $H_{\varepsilon}$, one breaks the chiral symmetry of the rest of the chain. For example, since the order parameter is a chiral 4-vector, the entrance of the chiral 4-vector into the Hamiltonian should be times another chiral 4 -vector. Therefore we might expect that $H_{\varepsilon}$ should be replaced by something which is just the 4-th component of a chiral 4-vector associated with the degrees of freedom at the new end of the chain, i.e. at $\eta=\varepsilon$. Although the whole chain is chirally symmetric, in the process of integrating out the degrees of freedom in the small $\eta$ region, an explicit chiral symmetry breaking for the rest of the chain has been introduced. The situation is very similar to an atomic impurity introduced into a ferromagnet. As a whole, the system

\footnotetext{
${ }^{4}$ Here we put in some numbers which have been omitted above.

${ }^{5}$ Note that they are UV only in the sense that in the LC frame it costs a lot of energy to disrupt them.
} 
is still rotationally symmetric. However, for most practical purposes the ferromagnet (without the impurity) can be regarded as frozen and the effective Hamiltonian for the impurity is not rotationally symmetric. The rotational multiplets are split and the spin of the atom precesses in the "external" field provided by the ferromagnet, thereby radiating off magnons. The time derivative of the angular momentum of the atom is equal to the amplitude for emitting a spin wave.

Now let us consider a specific scheme. What objects can one make out of quarks which transform like a chiral 4-vector. In order to simplify search for appropriate operators, it is useful to introduce the $\mathrm{SU}_{2} \times \mathrm{SU}_{2}$ "gamma" matrices (which have nothing to do with the true gamma matrices in the spin sense).

$$
\gamma_{\alpha}=\left(\begin{array}{cc}
0 & \tau_{\alpha} \\
\tau_{\alpha} & 0
\end{array}\right) \quad \gamma_{0}=\left(\begin{array}{cc}
0 & i \\
-i & 0
\end{array}\right)
$$

and $\gamma_{5}= \pm \sigma_{z}$, where the plus (minus) applies to quarks (antiquarks). The spin-flavor spinor of a quark in this basis has components $(u \uparrow, d \uparrow, u \downarrow, d \downarrow)$. These gamma matrices have the advantage that the indices here are chiral 4 -vector indices. The point is that possible objects for a one body operator, which transform like a chiral 4-vector, such as $\psi^{\dagger} \gamma_{4} \gamma_{\alpha} \psi$ do not commute with $\sigma_{z}$ and of course the chiral order parameter should commute with angular momentum. It is thus not sufficient to build the operator out of spin and isospin operators only, but one has to introduce some spin orbit coupling as well. ${ }^{6}$ One strange and peculiar fact when one goes to the LC frame is that chiral 4-vectors cannot be made out of one body operators. In the following, we will explicitly construct such an operator for the case of a two body system - a meson consisting of a quark and an antiquark (with LC momenta $\eta_{q}$ and $\eta_{\bar{q}}$ respectively). The most simple chiral 4-vector one can write for this system involves at least four angular momentum states: $|+\rangle,|-\rangle,|S\rangle$ and $|A\rangle$ which have the properties: $L_{z}|+\rangle=+|+\rangle, L_{z}|-\rangle=-|-\rangle$ and $L_{z}|S\rangle=L_{z}|A\rangle=0 .|S\rangle$ and $|A\rangle$ are symmetric and antisymmetric under interchange of $\eta_{q}$ and $\eta_{\bar{q}}$. Out of these operator one can now construct the 4-th component of a chiral four vector

$$
\phi_{4} \equiv\left[\left(\vec{\sigma}_{q}-\vec{\sigma}_{\bar{q}}\right) \times \vec{B}\right]_{z}
$$

where

$$
\begin{aligned}
& B_{-}=|S\rangle\langle+|+|-\rangle\langle S| \\
& B_{+}=|S\rangle\langle-|+|+\rangle\langle S| .
\end{aligned}
$$

The point is, Eq.(14) has spin orbit couplings between the quarks. Even though one can write down other candidates for a the 4-th component of a chiral 4-vector, all other candidates are more complicated.

\footnotetext{
${ }^{6}$ See also Ref.[4] for a more detailed discussion of this point.
} 
Table 1: Decay width for various meson decays involving $\pi$-emission.

\begin{tabular}{lcl}
\hline Decay & Computed width $(\mathrm{MeV})[3]$ & Expt. width $(\mathrm{MeV})[6]$ \\
\hline$a_{1}(1260) \rightarrow \sigma \pi$ & 47 & $<28$ \\
$a_{1}(1260) \rightarrow \rho \pi$ & 185 & $\lesssim 400$ \\
$\rho \rightarrow \pi \pi$ & 130 & 125 \\
$a_{2}(1320) \rightarrow \rho \pi$ & 24 & 16 \\
$a_{0}(980) \rightarrow \eta \pi$ & 75 & $?$ \\
$f_{1}(1285) \rightarrow a_{0}(980) \pi$ & 18 & $11\left[\right.$ ignoring $\left.a_{0} \rightarrow K \bar{K}\right]$ \\
$\sigma \rightarrow \pi \pi$ & 475 & $\sim 500$ \\
$f_{2}(1270) \rightarrow \pi \pi$ & 108 & 157 \\
$b_{1}(1235) \rightarrow a_{0}(980) \pi$ & 23 & $?$ \\
\hline
\end{tabular}

A number of physical consequences can be derived on the basis of these results (see Ref.[3] for a detailed discussion). For example, the (bare) pion should, together with its friend the (bare) $\sigma$, form a chiral 4-vector. Therefore they cannot be in an orbital angular momentum zero state! They are in an orbital angular momentum one state. This is quite surprising because one expects from the nonrelativistic quark model that the pion is in an s-wave - but that is simply not true in a LC frame. The (bare) $\rho$ and the (bare) $a_{1}$ form a chiral tensor. They can be in an s-wave. If one now adds $\phi_{4}$ from above (14), one discovers that the $\pi$ and the $a_{1}$ mix, while not affecting the $\rho$ and the $\sigma$. In a phenomenological model one can then calculate the wavefunctions of these hadrons by adjusting the symmetry breaking term to fit the hadron masses and one can then, in terms of these wavefunctions, calculate transition amplitudes. This has been done in Ref.[3] for the matrix elements of the chiral charge, which can then be used to calculate decay amplitudes involving the emission of pions.

The calculations were done in a very simple scheme in which one takes just 2 partons into account. Everything else is frozen. And the 2 partons simply interact with the rest of the frozen system. As the results in Table 1 show, with a few exceptions, one does pretty well - even with a minimal structure for the operators. In fact, considering that two quarks is a rather crude approximation, it is rather surprising how well the results fit the data. One can also use this scheme to understand some hadron masses.

The basic upshot one should get from these results is that one should think of these systems in a renormalization group way, where one first truncates the system to a small number of degrees of freedom and introduces an explicit breaking. Then one calculates physical observables and moves the cutoff back in a sequence of approximations - requiring that the physics remains invariant. This can also be translated into the statement that one must be looking for a fixed point of the renormalization group. 


\section{References}

1. L. Susskind, Phys. Rev. 165, 1535 (1968); see also J. Kogut and L. Susskind, Phys. Rep. 8C, 75 (1973), and references therein.

2. S. Weinberg, Phys. Rev. 150, 1313 (1966).

3. A. Casher and L. Susskind, Phys. Lett. 44B, 171 (1973).

4. A. Casher and L. Susskind, Phys. Rev. D9, 436 (1974).

5. K. Wilson et. al., Phys. Rev. D49, 6720 (1994).

6. Particle data group, Phys. Rev. D50, 1173 (1994). 